PACS: $62.20 . \mathrm{Fe}$

\author{
Yu.M. Podrezov
}

\title{
NANOCRYSTALLINE STRUCTURE FORMATION UNDER SEVERE PLASTIC DEFORMATION AND ITS INFLUENCE ON MECHANICAL PROPERTIES
}

Institute for Problems of Materials Sciences, NAS of Ukraine

3, Krzhizhanovsky Str., Kiev, 03142, Ukraine

Consideration is given to the distinction between nanocrystal strengthening and Hall-Petch grain size strengthening which varies linearly with $d^{-1 / 2}$. The important role of grain boundary structure in the strengthening formation of nanomaterials is emphasized. Static and dynamic recoveries are the main reasons limiting the minimal size of structural elements under deformation in high-deformed materials. Structural relaxation proceeding by the recovery mechanism during both the plastic deformation and unloading causes loss of strengthening in high-deformed materials. At the initial stage of repeated deformation the recovered cell structure interacts with moving dislocations in a special way. In the microdeformation level the hardening stress is practically independent of previous deformation degrees in a wide interval of deformation. The usual increase of strengthening with rise of deformation is observed only from the yield point.

\section{Introduction}

The creation of new high deformation methods offers ample scope for both the strain hardening theory and deformed materials structure engineering. Equalchannel angular pressing (ECAP) method was created by Segal [1]. Simple shear uniform deformation of high intensity can be achieved on a $15 \times 15 \times 150 \mathrm{~mm}$ macrosample without changing its sizes (Fig. 1). Another method based on the deformation by twist extrusion (TE) scheme was proposed by Beygelzimer [2] (Fig. 2). The creation of uniform shear deformation without size changing allows to carry out repeated deformed treatment in the different (or the same) direction of deformation and, as a result, to control structure formation process under high deformation degrees.

The achievement of high strength in deformed materials with nanostructure is essentially a more complicated task than it follows from the microscopic theory of strength. According to the models of this theory, strength of materials is related with structural element dimension by Hall-Petch equation $\sigma_{\mathrm{T}}=\sigma_{0}+k_{y} d^{-1 / 2}$ or its Holt variation for cells materials $\sigma_{\mathrm{T}}=\sigma_{0}+k_{y} d^{-1}$. The extrapolation to nanoscale of 

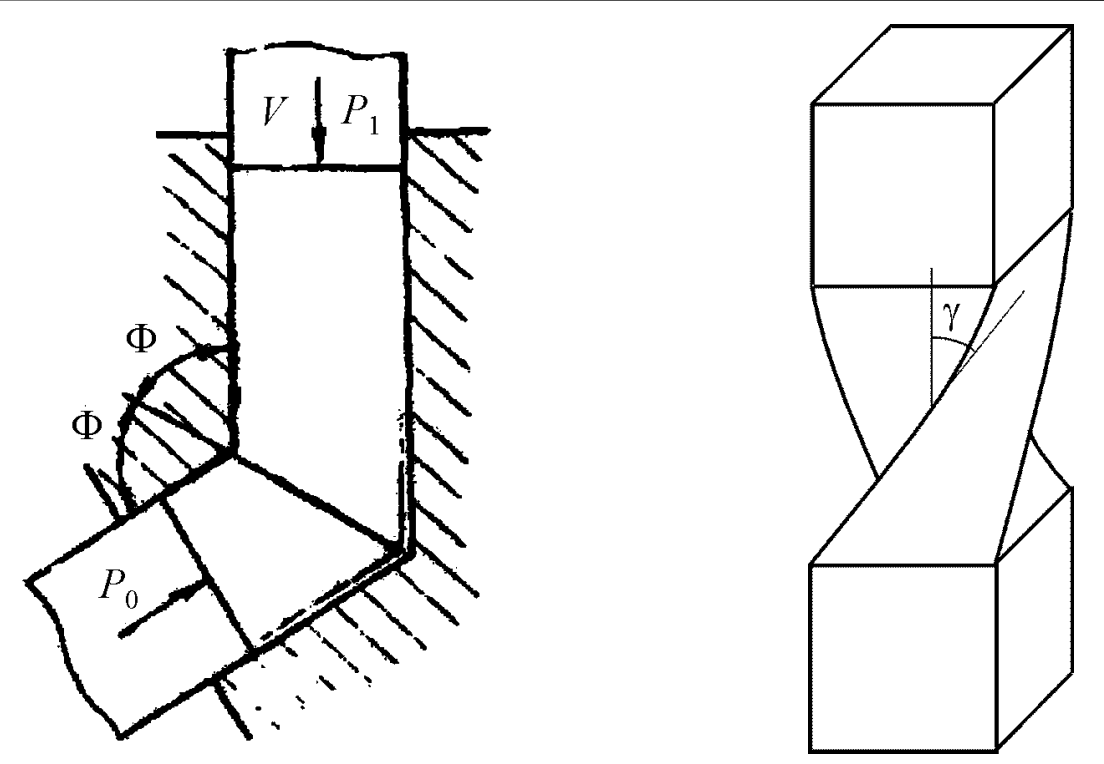

Fig. 1. ECAP scheme [1]

Fig. 2. Deformation by TE [5]

grain or cell dimension theoretically predicts a very high strength of nanocrystalline materials. But experimental results demonstrate essentially worse situation. A.W. Tompson [3] reviewed experimental data from variety of investigations obtained on high-deformed armco-Fe wires. Relation between flow stress increment $\sigma-\sigma_{0}$ and substructure size is shown in Fig. 3. In this graph we add our data obtained on armco-Fe deformed by ECAP.

Important conclusions about interrelation of structural evolution and strengthening of high-deformed materials follow from these results. Firstly, it is the change of strengthening mechanism from grain size sensitivity (Hall-Petch equation) to cell size sensitivity (Holt equation) at critical grain size $d<0.4 \mu \mathrm{m}$. Sec-

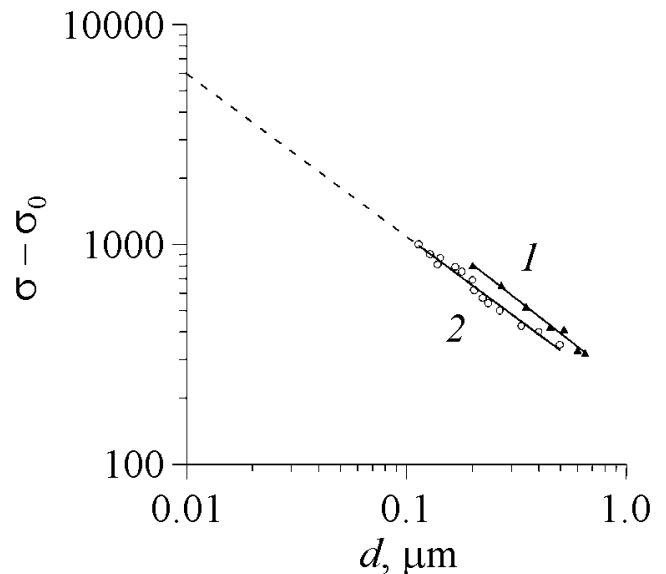

Fig. 3. Effect of cell size on yield point of high-deformed armco-Fe after ECAP (1) and wire drawing (2) [4] ondly, it is the theoretical possibility to obtain superhigh strengthening in nanocrystalline armco-Fe. It follows from experimental data (see dashed line) that extrapolation of experimental data to nanostructure sizes $(10 \mathrm{~nm})$ gives, for such material, the value of yield point of approximately $6000 \mathrm{MPa}$. But the thread conclusion restricts such possibility. Difficulty is amplified due to the fact that the cell or subgrain size in armco-Fe obtained by different methods of severe plastic deformation (rolling, drawing, EC-pressure) cannot be less than 100 $\mathrm{nm}$. As a result, the yield point for such material is only $1000 \mathrm{MPa}$ [3-6]. 


\section{Experimental procedure}

In order to explain the restriction of structural dispersion in deformed materials careful investigations of deformed substructure by TEM were carried out. More important experimental work was published by Langford and Cohen [6] who investigated highdeformed armco-Fe wires. Practically the same results were obtained in our work, where severe plastic deformation in armco-Fe was produced by rolling and ECAP.

\section{Results and discussion}

The following expression for $f$, the fraction of the initial number of cells in a cross section at any given deformation power can be written

$$
f=\frac{N}{N_{\text {in }}} \exp \left[-\left(e-e_{\text {in }}\right)\right],
$$

where $N_{\text {in }}$ is the initial number of cells per unit cross-sectional area as formed at some early strain $e_{\text {in }}$ and $N$ is the number of cells per unit cross-sectional area at some subsequent strain $e$. Measurements of $N$ have been made during the deformation and corresponding values of $f$ versus $e$ are plotted in Fig. 4. As it can be deduced from Fig. 4, $f$ is near unity both for wire drawing and for rolling deformation under low and medium deformation degrees. This result is in a good agreement with the Tailor-Pallany law: both bulk material and structural elements (grains, cell) have the same shape under deformation. Since ECAP is a process capable of producing plastic deformation without causing substantial change in geometric shape of billet, the cell size of ECAP deformed iron at the medium deformation degrees is substantially higher.

Under high deformation power principal changing in $f(e)$ dependence takes place. There is the large decrease in $f$ throughout the subsequent elongation, signifying that substantial dynamic recovery is operative during the cell refinement. This means that many cells are being lost from the structure simultaneously with

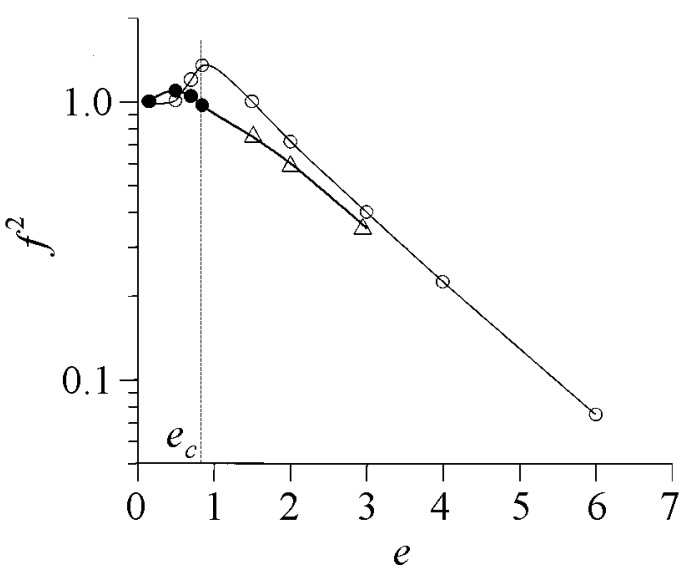

Fig. 4. The dependence of $f$-parameter on deformation degree $e: \bullet-$ rolling, $\Delta-\mathrm{ECAP}$, $\mathrm{o}$ - wire drawing [4] cross-sectional reduction of the remaining cells. It is clear that cell wall migration constitutes a very important aspect of the structural changes and the critical deformation degree $e_{c}$, under which changing in $f(e)$ dependence takes place, is a very important magnitude. Cell size evolution under deformation is accompanied by the increasing of cell misorientation.

According to Tompson's model [3] of structural evolution, static and dynamic recoveries are the main reasons limiting the minimal size of structural elements under deformation in high- 
deformed materials. On one hand, many cells are being lost from the structure during this process, on the other hand, the recovery process promotes boundary perfection and increases misorientation of cells. But this model cannot explain the existence of critical deformation degree $e_{c}$, which characterizes start or strong activation of this process.

Rybin [7] proposed the disclination model of severe plastic deformation. This model is based on the analysis of micromechanical stress arising around a dislocation during plastic deformation. According to this model, plastic deformation in crystals is developed by moving dislocation at a low and medium deformation. But after transformation of substructure from dislocation forest to cells at $e_{i} \sim 0.2$ a change of deformation mechanism takes place and severe deformation is evoked by generation of disclination modes without diminution in subgrain size.

Calculation in the framework of disclination model gives critical cell size $\sim 0.2 \mu \mathrm{m}$ for BCC metals. As far as the critical cell size depends on some physical constants (Burgers vector, elastic modulus, stacking fault energy), for some FCC metals its value is essentially lower. It is in a good agreement with recent experimental data, obtained on ECAP deformed $\mathrm{Ni}$ and $\mathrm{Al}$ where the average size of structural element was 30-50 $\mathrm{nm}$ [8]. The disclination theory predicts monotonic rise of misorientation with the increasing of deformation power and does not imply any spatial features of mechanical behaviour of high-deformed materials after critical deformation $e_{c}$.

Near the critical deformation $e_{c}$ there is the change in the mechanical behavior of materials [9]. The parabolic strain hardening which was observed at the low and medium deformation degrees was followed by the linear stage of deformation at high deformation degrees. The growth of the fracture toughness for specimens

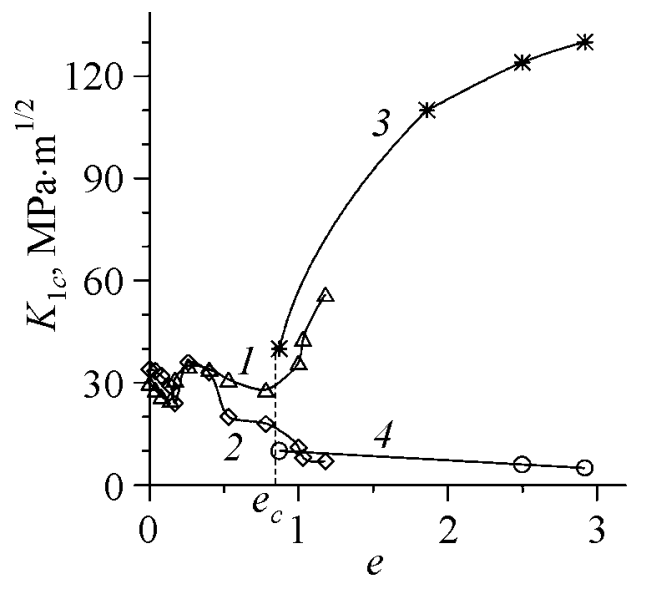

Fig. 5. Fracture toughness of strained iron: 1,2 - rolling; 3, 4- $\operatorname{ECAP}(1,3-$ crack introduced into the plane perpendicular to the plane of deformation; 2,4 - crack introduced into the plane parallel to the plane of deformation) with cracks introduced into the plane perpendicular to the plane of deformation was shown.

Mechanical behavior near critical deformation $e_{c}$ was observed in highdeformed armco-Fe after ECAP. The growth of the fracture toughness for specimens with cracks introduced into the plane perpendicular to the plane of deformation was shown, and the decreasing of the fracture characteristic for specimens with cracks introduced into the plane parallel to the plane of deformation was observed (Fig. 5). The increasing of the deformation degree $\left(e>e_{c}\right)$ promotes the change of the failure mechanism as: quasi-cleavage $\rightarrow$ quasi-cleavage with delamination. On the contrary, transition 
from the parabolic stage of strain hardening to the linear one is not related with the change of the deformation mechanism. Therefore the processes occurring near critical deformation $e_{c}$ are very important and must be investigated more carefully.

The noted features of structure formation in deformed materials predetermine regularities of designing their mechanical properties. Analysis of strengthening curves for a deformed material is made usually basing on structural changes occurring in the material at its continuous loading. And it is assumed that each successive structural condition follows from the evolution of the previous one, and a change of the deforming stress is a consequence of the evolution. Structure sensitive models of strain hardening have been described in detail by many authors [10-12].

Practically the same approaches are used to analyze structural sensitivity of mechanical properties of prestrained materials [5]. Widely used is the known postulate of mechanics that deforming stress being achieved in a material at repeated deformation corresponds exactly to deforming stress achieved in it at the unloading moment at initial deformation. This postulate does hold well in the range of low and medium deformation degrees for materials which have not susceptibility to recovery process [13]. For example, it was verified enough really in $\mathrm{Al}-\mathrm{Ti}-\mathrm{Cr}$ intermetallic specimens in our experiments under uniaxial compression tests, Fig. 6,a. But this postulate does not hold for materials which have inclination to recovery. Such results were obtained in aluminium (Fig. 6,b) which is inclined to recovery. Strain hardening curve obtained by single loading compression test (Fig. 6, $b$, curve 1 ) was compared with curves obtained after repeated deformation (second deformation curve 2, third deformation - curve 3 and fourth deformation - curve 4). Experimental data show that prestrained materials have both lower yield point and lower strain hardening coefficient than it follows from the postulate.

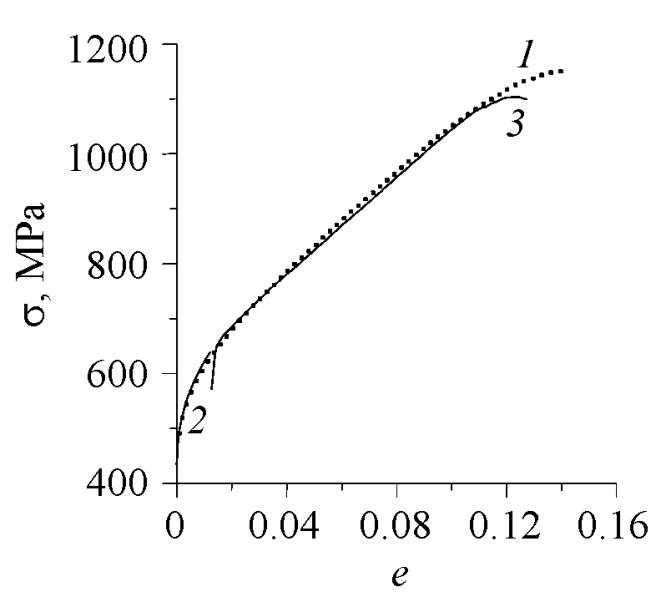

$a$

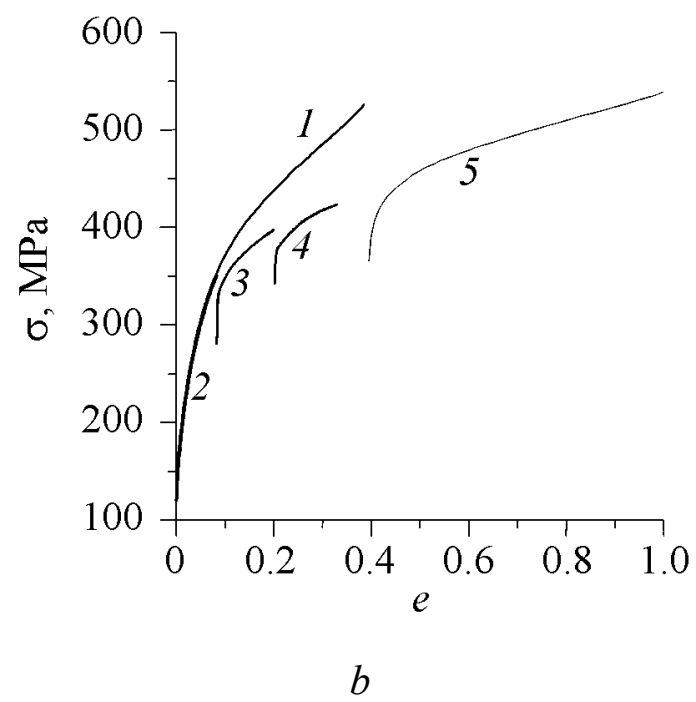

Fig. 6. Stress-strain curves of Al-Ti-Cr alloys $(a)(1,2$ - continuous deformation, 3 repeated deformation) and of pure $\mathrm{Al}(b)$ (1, 2-continuous deformation, 3-5 - repeated deformation) 
Unfortunately, due to the fact that conditions of uniaxial deformation are limited by friction and size effects under compression and by neck formation process under tension, it is impossible to verify the postulate at high deformation powers (more than $e=0.4$ ). Therefore, in order to obtain strain hardening curve of highdeformed aluminium in unified loading condition we have made repeated grinding of lateral faces of a specimen deformed by single compression. As a result, the specimen was put into shape of rectangle and tested by repeated compression. This strain hardening curve (curve 5) lies essentially lower than single one (curve 1) but its start position coincides with the end of last curves obtained by repeated compression (curve 4). Since the summary hardening curve of predeformed materials could be obtained by mating results of consecutive loading cycles, but because of recovery process its strain hardening coefficient may be essentially lower than one obtained under continuous loading.

In titanium specimens we obtained similar effects [14]. Strain hardening curves obtained by uniaxial tension (subject to neck formation moment) and data on hardening of the material after high-deformed rolling were compared (Fig. 7). In this case, the hardening is stronger essentially for the specimens tested in continuous loading than that for the specimens tested in reloading of predeformed material. In particular, failure stress of $1600 \mathrm{MPa}$ is achieved under uniaxial tension of pure titanium. In high-deformed rolled titanium the stress never exceeds 600-700 $\mathrm{MPa}$. Undoubtedly, a difference of both loading conditions and of specimen texture should be taken into account in this case. However only these factors do not in any case explain essential difference in titanium properties in conditions of continuous loading and pre-deformation states.

Larikov [15] explains the recovery process by increasing the mobility of screw dislocation components and, as a result, activation of cross slip in plastic deformation mechanism. The processes of structural relaxation proceeding by recovery mechanism both during plastic deformation and during unloading seem to be the most important factors to promote loss of strengthening in high-deformed materials.

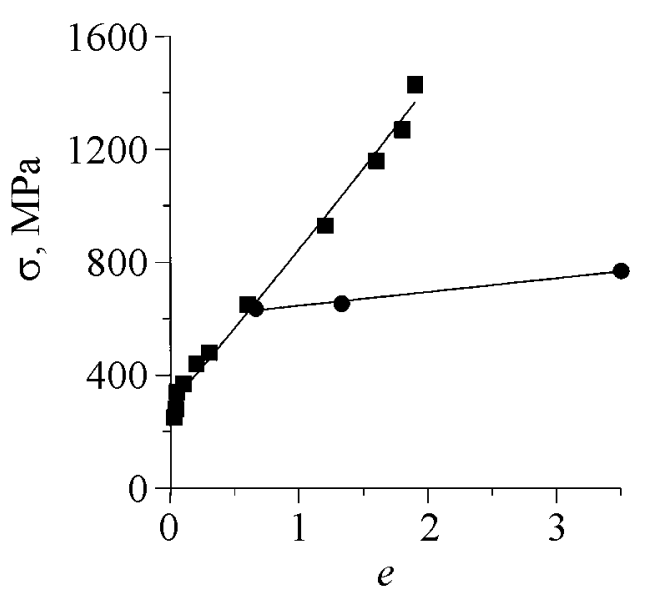

Fig. 7. Stress-strain curves for Ti: tension, $\bullet-$ rolling

We analyzed microplasticity curves of predeformed rolled molybdenum specimens (range of deformation degrees 9-75\%). It was found (Fig. 8) that at the initial stage of repeated deformation microplasticity stress-strain diagrams are insensitive to pre-deformation degrees (and therefore to structural state of deformed materials). In the microdeformation level at $10^{-5}, 10^{-4}$ and $5 \cdot 10^{-4}$ deformation degrees (Fig. 9) the deformation stress is practically the same for all investigated materials. Differences start to be noticeable at $2 \cdot 10^{-3}$ (yield point). And only 


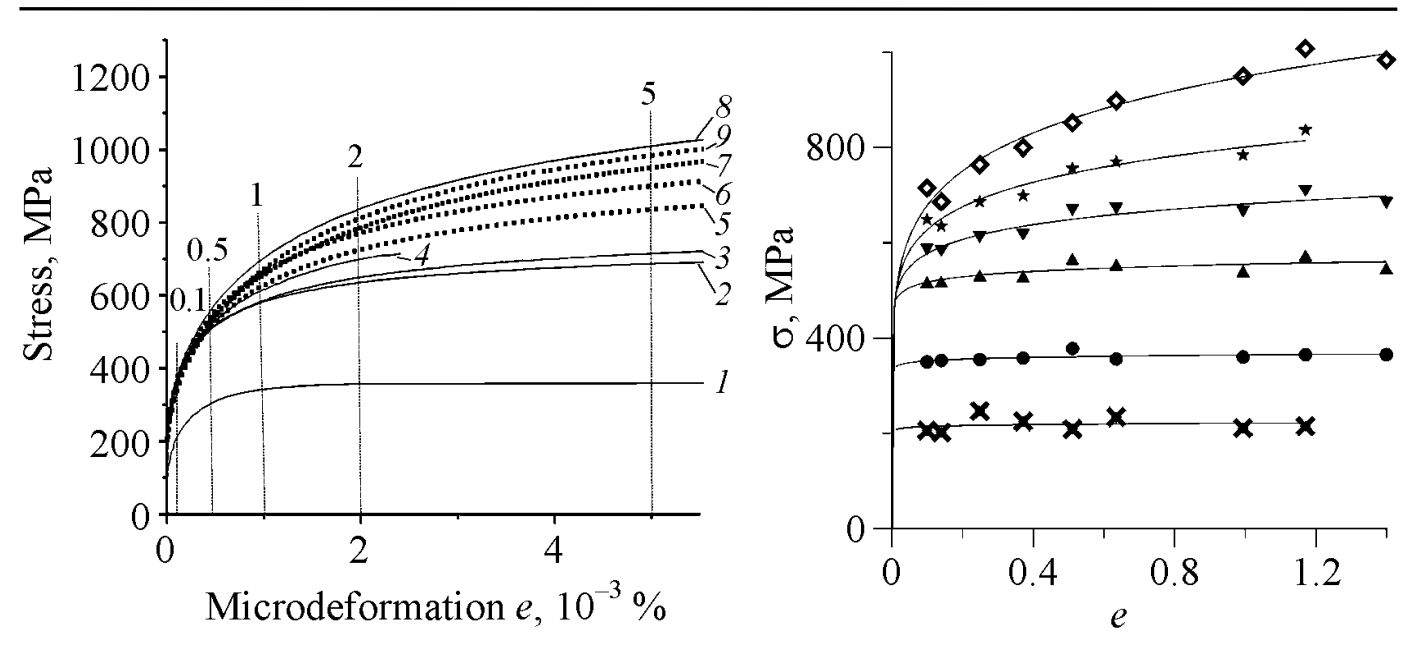

Fig. 8. Microplasticity curves of molybdenum deformed by rolling to different deformation degrees, \%: 1 - initial, $2-9,3-13,4-23,5-39,6-53,7-63,8-68,9-73$

Fig. 9. Stress-prestrain dependences of molybdenum deformed by rolling for different microplasticity levels: $\diamond-5 \cdot 10^{-3}, \star-2 \cdot 10^{-3}, \mathbf{\nabla}-1 \cdot 10^{-3}, \mathbf{\Delta}-5 \cdot 10^{-4}, \bullet-1 \cdot 10^{-4}, \times-1 \cdot 10^{-5}$

at comparatively high repeated deformation degree $5 \cdot 10^{-3}$ the stable increase of stress with the rise of deformation is observed. Obviously, the hardening curves obtained at microdeformation levels cannot be explained in the framework of traditional structural model. In the initial stage of repeated deformation the recovered cells structure interacts with the moving dislocation in a spatial way.

These data are important for creation of an adequate model of mechanical behaviour of high-deformed materials, and may be useful in the explaining of anomalous dependence of fracture toughness on deformation power of materials tested in ductile-brittle transition temperature range (see Fig. 5). In this case, the laws of interaction of dislocations being emitted from a crack tip with primary structure, in conditions when number of the dislocations is not too large, should be accounted for.

\section{Conclusions}

Cells or subgrain size in armco-Fe obtained by different methods of severe plastic deformation (rolling, drawing, EC-pressure), cannot be less than $100 \mathrm{~nm}$. As a result, the yield point for such material is only $1000 \mathrm{MPa}$. Static and dynamic recovery are the main reasons limiting the minimal size of structural elements under deformation in high-deformed materials.

The change of mechanical behavior near critical deformation $e_{c}$ was observed in high-deformed armco-Fe after ECAP .The growth of the fracture toughness for specimens with cracks introduced into the plane perpendicular to the plane of deformation was shown, and the decreasing of the fracture characteristic for specimens with cracks introduced into the plane parallel to the plane of deformation was observed. Increasing of the deformation degree $\left(e>e_{c}\right)$ promotes the change of the failure mechanism as: quasi-cleavage $\rightarrow$ quasi-cleavage with delamination. 
Postulate of mechanics, that deformation stress being achieved in a material at repeated deformation corresponds exactly to deformation stress achieved in it at unloading moment at the initial deformation, does hold well for materials which have no susceptibility to recovery process. But this postulate does not hold for materials which have inclination to recovery. Structural relaxation proceeding by recovery mechanism both during plastic deformation and during unloading promotes loss of strengthening in high-deformed materials.

In the initial stage of repeated deformation the recovered cells structure interacts with the moving dislocation in a spatial way. In the microdeformation level, the hardening stress is practically independent of previous deformation degrees in the wide interval of deformation. Usual increase of strengthening with rise of deformation is observed only starting from the yield point $(e=0.002)$.

The author wishes to thank Prof. S. Firstov and Dr. M. Danylenko for fruitful discussion. I also thank Dr. D. Verbylo who assisted in the experiments.

1. V.M. Segal, V.I. Reznikov, V.I. Kopilov, D.A. Pavlik, V.F. Malyshev, Processes of Plastic Structural Formation of Metals, Nauka i technika, Minsk (1994) (in Russian).

2. Y. Beygelzimer, V. Varyukhin, D. Orlov, B. Efros, A. Salimgareyev, V. Stolyarov, U1trafine Grained Materials: Processing and structure, Washington (2002), p. 137-142.

3. A.W. Tompson, Met. Trans. 8A, 833 (1977).

4. G. Langford, M. Cohen, Trans. ASM 62, 623 (1966).

5. R.C. Clenn, G. Langford, A.S. Keh, ASM Trans. Quart. 62, 285 (1969).

6. G. Langford, M. Cohen, Met. Trans. 6A, 901 (1975).

7. V.V. Rybin, Prob. Mater. Sci. 29, 11 (2002) (in Russian).

8. N.I. Noskova, Prob. Mater. Sci. 29, 309 (2002) (in Russian).

9. Yu.N. Podrezov, N.I. Danilenko, V.I. Kopylov, S.A Firstov, FTVD 11, № 2, 33 (2001).

10. J. Bell, Experimental Bases of the Mechanics of Deformed Bodies, Part 1, London (1984).

11. V.I. Trefilov, V.F. Moiseyev, A.P. Pechcovsky, Work Hardening and Fracture of the Polycrystal Metals, Trefilov V.I. (Ed.), Naukova Dumka, Kiev (1975) (in Russian).

12. M.J. Zehetbauer, Ultrafine Grained Materials: Processing and structure, Washington (2002), p. 39-57.

13. Yu.V. Milman, D.B. Miracle, N.P. Korzhova, Yu.N. Podrezov, Intermetallics 9839 (2001).

14. Yu.N. Podrezov, M.V. Minakov, Electron microscopy and strength of materials № 9, 60 (1998) (in Russian).

15. L.N. Larikov, Healing of defects in metals, Naukova Dumka, Kiev (1980) (in Russia). 